Brit. Heart F., 1968, 30, 679.

\title{
Vascular Pattern of Myocardium of Right Ventricle of Human Heart
}

\author{
GEOFFREY FARRER-BROWN ${ }^{\star}$
}

\author{
From the Pathology Department, Northwestern University Medical School, Chicago, Illinois, U.S.A., and the \\ Bland-Sutton Institute of Pathology, Middlesex Hospital Medical School, London W.1
}

Apart from comparisons of the density of the blood supply of the right and left ventricles in various age-groups (e.g. Gross, 1921; Campbell, 1929; Whitten, 1930a, b; Ehrich, de la Chapelle, and Cohn, 1931; Gross and Kugel, 1933), the arterial pattern of the myocardium of the right ventricle of the human heart has, in the past, received little attention. More recently, Mitchell and Schwartz (1965) and Fulton (1965) have published radiographs of the blood supply of both ventricles, but except for Fulton's description of the anastomotic vessels these investigators did not comment on the normal detailed vascular anatomy of the right ventricle. This paper describes the vascular pattern of the right ventricle seen in a study of a series of human hearts, $\dagger$ and discusses the possible importance of the left coronary arterial tree in the supply of the right ventricle.

\section{Material AND Methods}

Details of the hearts studied, the methods used to inject the coronary arteries with Chromopaque, to take microradiographs of transverse ventricular slices onto Kodak High Resolution plates, and the gross and microscopical examination of each heart have already been. published (Farrer-Brown, 1968a, b).

\section{ResUlts}

In 49 of the 52 hearts studied the right coronary artery supplied all of the right ventricular free wall, except the anterior margin which was supplied by branches of the left anterior descending artery: the right posterior descending artery supplied the posterior wall and the right marginal artery the lateral

Received December 29, 1967.

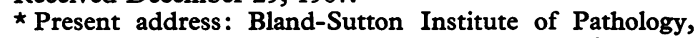
The Middlesex Hospital Medical School, London W.1.

$t$ The results of this study, as a whole, have been presented in the form of an M.D. thesis (Farrer-Brown, 1967). wall. In the other 3 hearts the artery in the posterior interventricular groove was a branch of the left circumflex artery, and consequently some of the posterior margin of the right ventricular wall was supplied by branches from this artery.

Because the muscle mass of the right ventricle was smaller than that of the left ventricle, the vascular supply was less conspicuous and appeared less abundant, but the basic pattern was the same as in the left ventricular free wall.

The branches from the main coronary arteries on the epicardial surface coursed directly through the myocardial wall towards the endocardium. Soon after entering the myocardium these "branching" type arteries (Farrer-Brown, 1968b) divided in a cascading tree-like pattern, giving off many small branches of gradually diminishing calibre.

In addition to these "branching" arteries there were "straight" type arteries, which gave off only a few twigs and maintained their calibre as they passed through the wall to supply the trabeculae carneae and papillary muscles. Fig. 1 shows the appearance of both these types of vessels in a midventricular transverse slice of an adult heart. A microradiograph of a myocardial slice cut slightly tangentially emphasizes well the presence of this arterial pattern (Fig. 2). Since the slices of the infants' hearts contain relatively more vessels than an equivalent thickness of an adult human heart the microradiographs of the former show the pattern extremely well (Fig. 3). The only exception to this pattern was anteriorly and posteriorly at the junction with the interventricular septum, where the branches of the anterior and posterior descending coronary arteries ran in a more circumferential direction.

In those hearts in which the capillaries had been injected the microradiographs showed that in the right ventricle these vessels ran alongside the muscle fibres, and that the appearance was similar to that of the left ventricle (Fig. 4). 


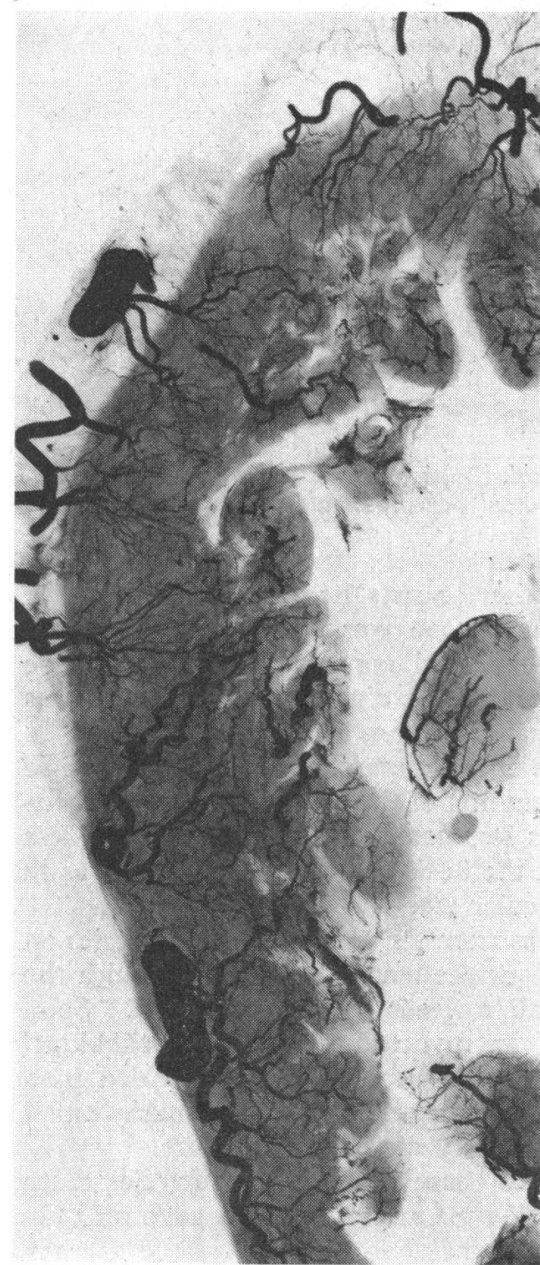

FIG. 1.

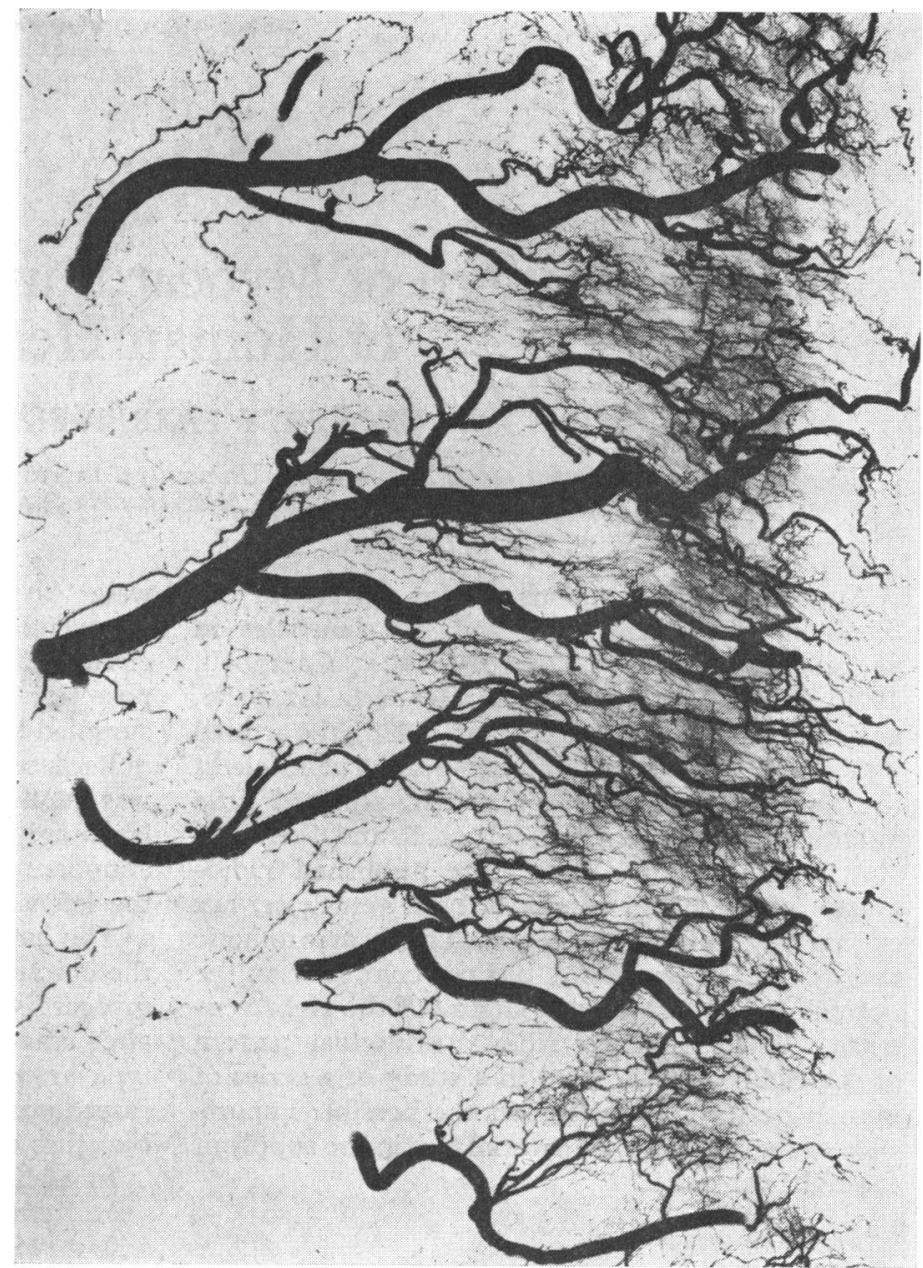

Fig. 2.

Fig. 1.-The normal arterial pattern of the right ventricle of the adult heart. $(\times 3$.

Fig. 2.-The right ventricle of an adult heart cut slightly tangentially to emphasize the similarity in arterial pattern compared to the left ventricle. $(\times 4$.

Papillary Muscles. The muscle columns that form the papillary muscles in the right ventricle are supplied by "straight" type arteries. The arteries supplying the anterior papillary muscle arose both from branches of the right coronary artery and the left anterior descending artery. The posterior papillary muscle was supplied by branches from the posterior descending artery, but frequently it also received a supply from the terminal branches of the septal branch of the left anterior descending artery when this artery supplied over 60 per cent of the interventricular septum, as illustrated in a midventricular slice of the heart of an infant of 2 weeks in age (Fig. 5). In this heart a vessel was seen to pass across the posterior papillary muscle connecting a terminal branch of the septal branch of the left anterior descending artery with a right coronary artery branch on the postero-lateral wall of the left ventricle (Fig. 6). The continuity of this vessel was confirmed by clearing the heart slice and dissecting out the artery under a dissecting microscope. The "straight" type arteries coursed through the middle of the papillary muscle giving off branches to the periphery (Fig. 7), in a manner similar to that seen in the papillary muscles of the left ventricle (Farrer-Brown, 1968b).

Moderator Band. Fig. 8 illustrates a large branch from the left anterior descending artery that passes along the length of the moderator band in the right 


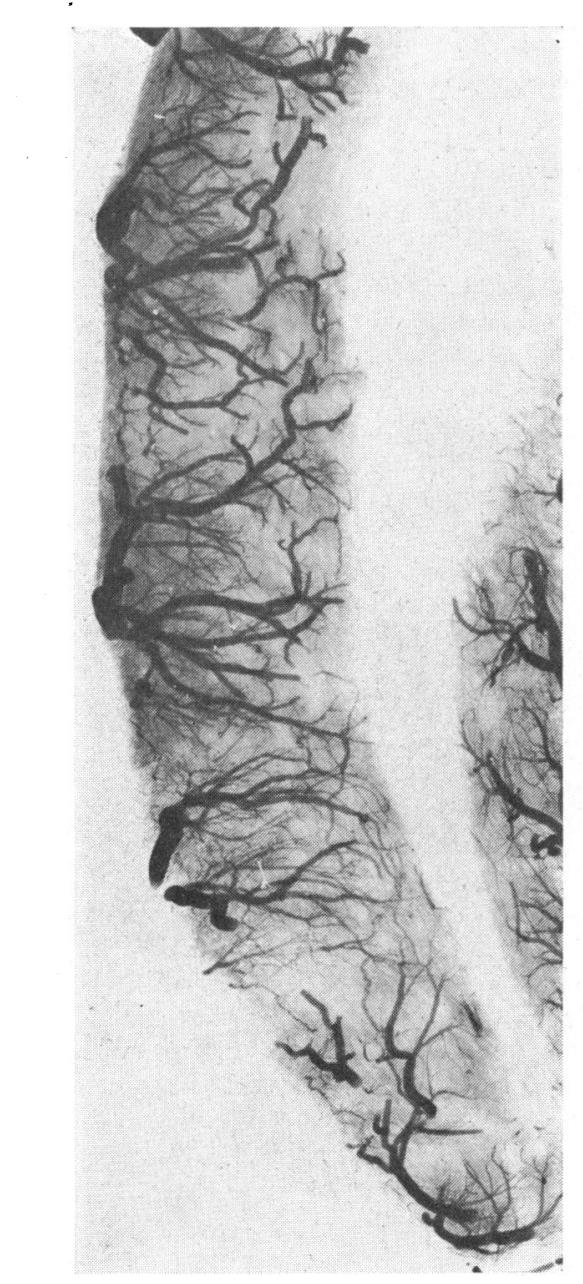

FIG. 3.-The right ventricular wall of a 2-week-old infant also showing the basic arterial pattern. $(\times 5 \cdot 6$. $)$

ventricle. This artery, which measured up to $1000 \mu$ in diameter in hearts with prominent moderator bands, may constitute an important part of the supply of the anterior papillary muscle of the right ventricle. It may also supply blood to the antero-lateral part of the right ventricular free wall, as was also found in the heart of an infant (Fig. 5). The continuity of this artery was also confirmed by dissection.

Other Anastomotic Vessels in the Right Ventricle. The technique of injection used in this study was not designed to prove the existence of anastomotic vessels in the heart nor to estimate their number or size. It is, however, relevant to note the location of anastomotic arteries seen within the myocardial

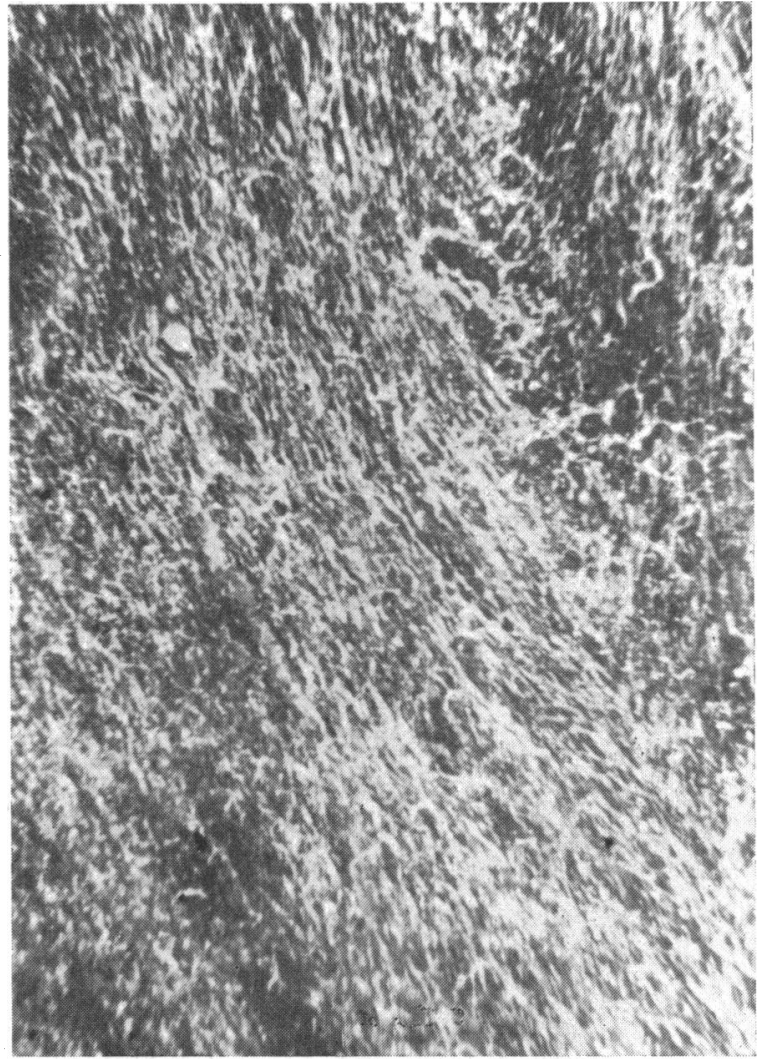

Fig. 4. - Capillaries in the right ventricle. $(\times 30$.

wall of the right ventricle of hearts showing severe coronary artery disease. Anastomotic arteries were seen in the subendocardial zone (Fig. 9), in the outer half of the posterior wall (Fig. 10), across the anterior and posterior papillary muscle columns, and in the anterior papillary muscles between left and right coronary artery trees (Fig. 11).

\section{Discussion}

This study has shown that the pattern of arteries in the right ventricle is basically the same as that seen in the left ventricle, which might be expected from consideration of the development of the heart. The fact that the vessels are less conspicuous appears to be due to the smaller muscle bulk of this ventricle.

Gross (1921) and Gross and Kugel (1933) suggested that with increasing age there was a relative decrease of the vascularity of the right ventricle compared to the left, but this was not observed in the present study, and the right ventricles of 2 


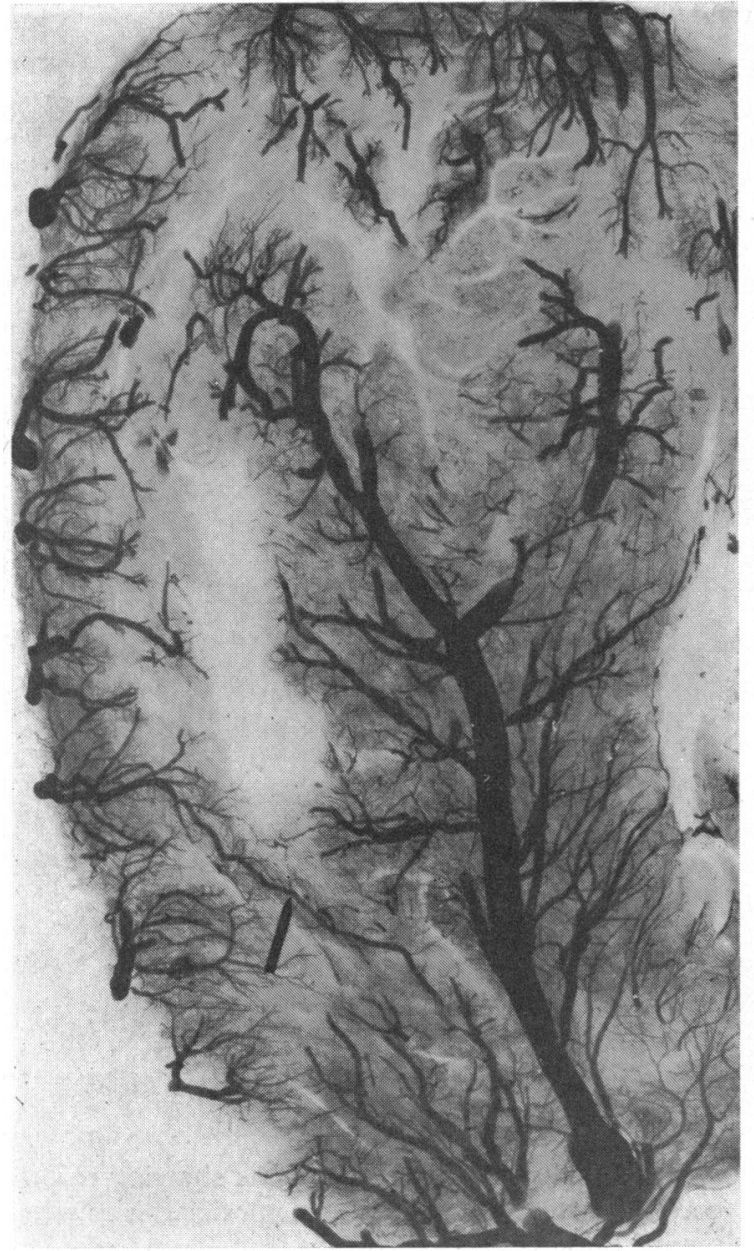

FIG. 5.-A septal branch of the left anterior descending artery supplying the posterior papillary muscle in the heart of a 2-week-old infant. Note also the artery running along the moderator band and communicating with the left ventricular free wall (arrowed). ( $\times 5$.)

infants, each 2 weeks of age, showed a similar pattern of blood supply to that of adult hearts.

There are probably two reasons why the present observations differ from those of Gross. First, in this study, account was taken of the relatively greater number of vessels that are present in the transverse slices of infants' hearts when these slices are of similar thickness to the adult hearts. Secondly, the magnification achieved, and, in particular, the enlargements of the microradiographs of the hearts of the infants up to the same size as the adult hearts, allowed a more accurate comparison.

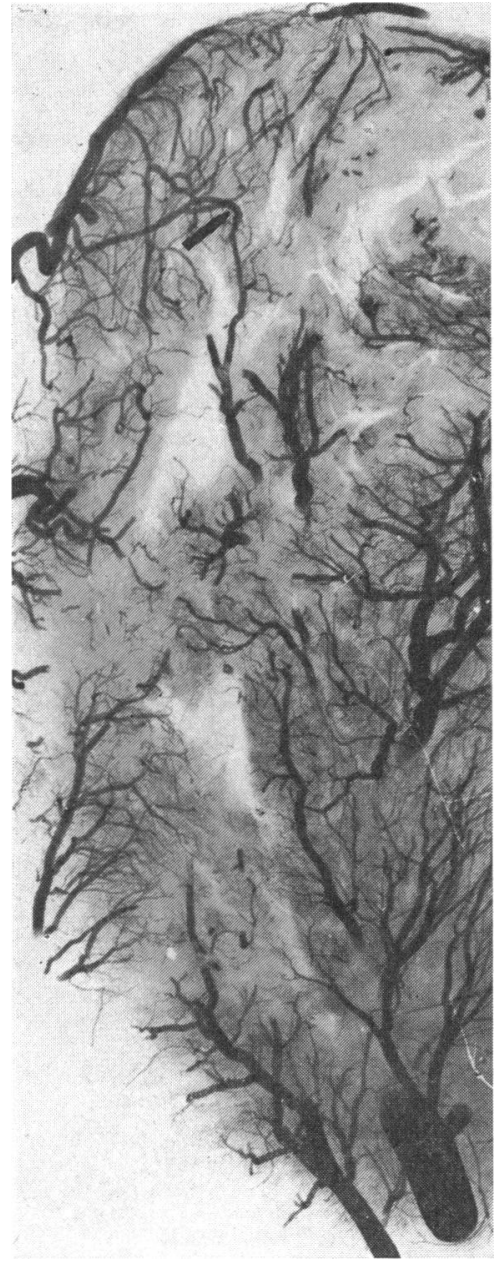

FIG. 6.-A communicating vessel is present between the branches of the right marginal artery and a septal branch of the anterior descending artery in the heart of a 2-week-old boy. $(\times 5$.

The radiographic observations have suggested that the left coronary artery may provide a substantial blood flow to areas of the right ventricle in normal hearts. The large artery in the moderator band, first mentioned by Campbell (1929) and also seen by Fulton (1965), was found to be consistently present and measured up to $1000 \mu$ in diameter. It appears to provide a major blood supply to the anterior papillary muscle of the right ventricle, and has been shown in this study to exist in the heart of an infant ( 2 weeks old) and communicate with the lateral wall of the right ventricle even at this early age. 


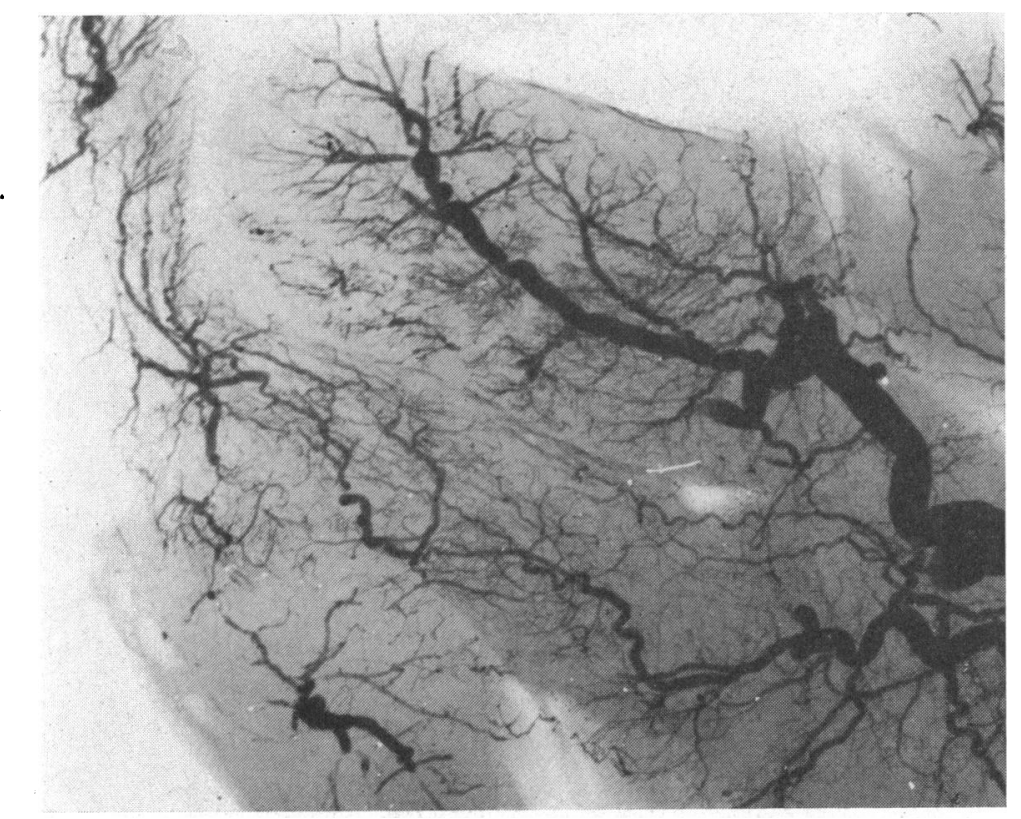

Fig. 7.-The arterial pattern within the anterior papillary muscle of the right ventricle. $(\times 10$.

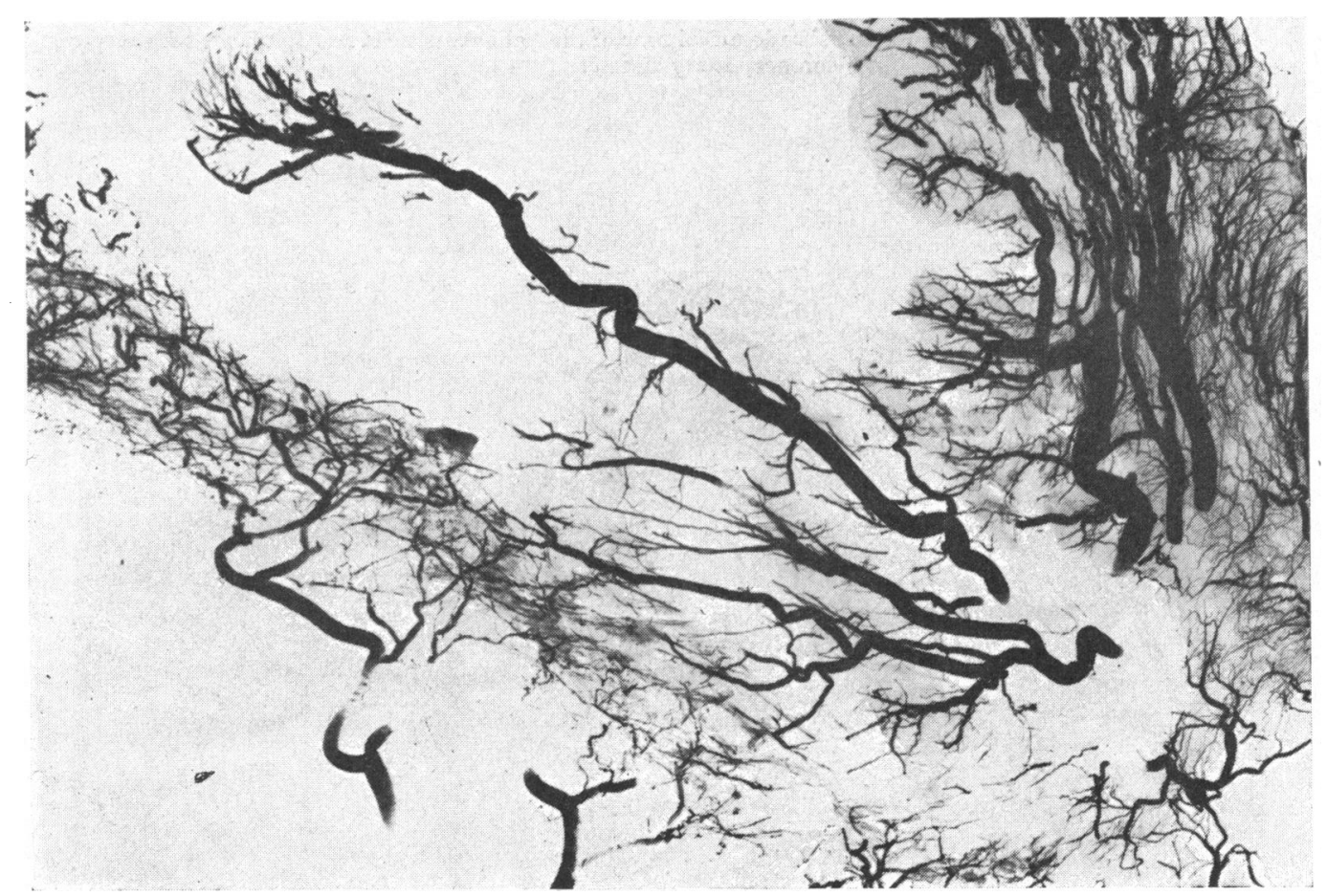

Fig. 8.-A large branch from the anterior descending artery running in the moderator band of the right ventricle. $(\times 3 \cdot 5$. 


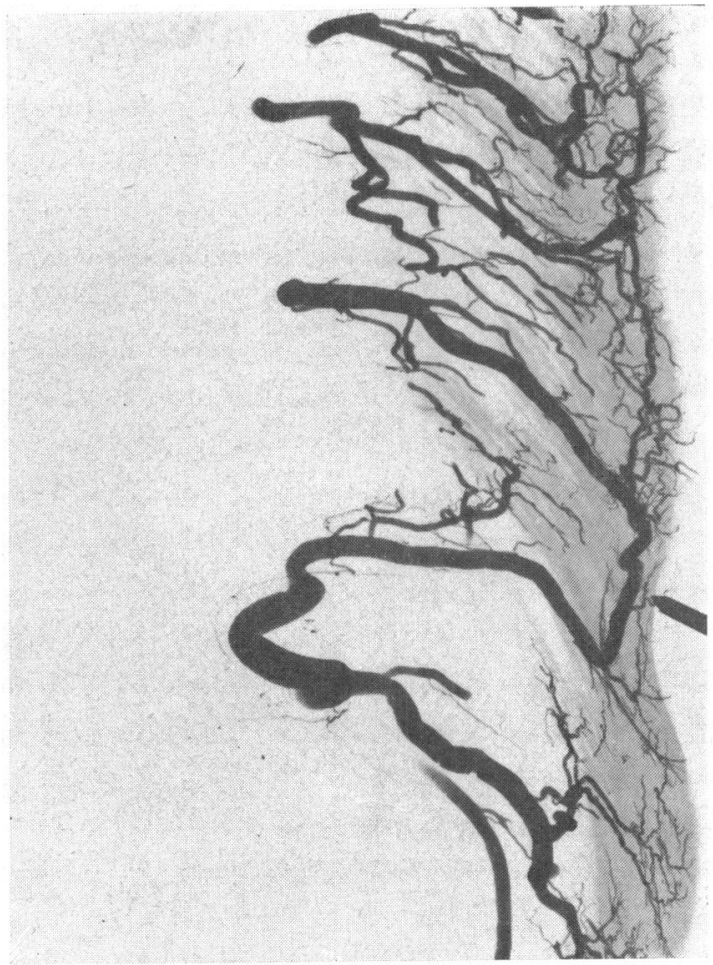

FIG. 9. - An anastomotic artery in the subendocardial zone of the right ventricle in an adult heart with severe coronary artery disease. $(\times 4$.

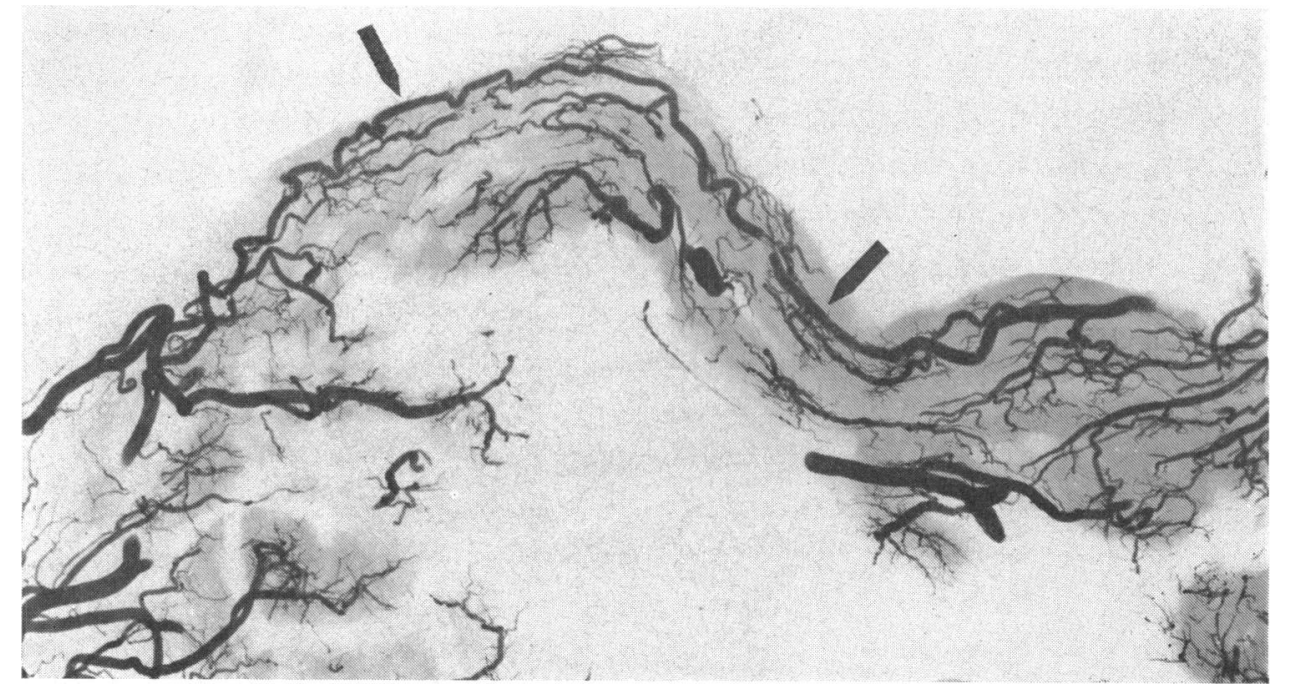

Fig. 10.-An anastomotic vessel between the branches of the right posterior descending and right marginal arteries in an adult heart with severe coronary artery disease. $(\times 4 \cdot 3$.) 
A proportion of the anterior margin of the right ventricle is also supplied by branches from the left anterior descending artery. In the majority of hearts, the terminal branches of the left anterior descending artery supply the posterior right ventricle at the apex as this artery terminates on the posterior wall. The mid-ventricular posterior margin of the right ventricle is occasionally supplied by the left coronary tree when the left circumflex artery provides the main posterior or interventricular artery.

Another important source of blood from the left coronary tree goes to the right posterior papillary muscles via the mid-septal branches of the anterior descending artery which may be present when this artery supplies over 60 per cent of the interventricular septum.

Vessels from the left coronary artery supply the right ventricle via arteries in the anterior margin of the free wall, the moderator band, the apex, and sometimes the interventricular septum and the posterior papillary muscles. Vessels of between 100 and $300 \mu$ diameter that appear to be anastomotic have been seen in these areas in hearts showing severe coronary artery disease. It seems probable that a much more extensive flow of blood than has previously been realized passes from the left coronary artery into the right ventricle in times of need, such as in generalized severe coronary artery disease or right coronary artery occlusion.

It has already been mentioned that only occasionally do branches from the left circumflex artery supply the posterior margin of the right ventricle in the mid and upper ventricular areas. Consequently, this posterior area of the right ventricular wall could be more prone to ischaemic damage in the event of occlusion of the right coronary artery tree than the remainder of the right ventricular wall, since the latter has a potential alternative blood supply from the left coronary artery, as described above. This idea is supported by the findings of Wade (1959) who, in a survey of 20 isolated cases of right ventricular infarction, found that the major lesions were confined to the posterior wall.

An additional factor favouring the right ventricle in respect of its blood supply may be the relative abundance of Thebesian vessels in the right ventricle compared with the left (Horn et al., 1950). However, no evidence exists yet as to the amount of nutrition the muscle fibres of the right ventricle obtain via these vessels. The normal blood supply via the arteries and capillaries is likely to be of much greater importance.

\section{SUMMARY}

The vascular pattern of the right ventricle seen in a series of 52 human hearts studied at necropsy

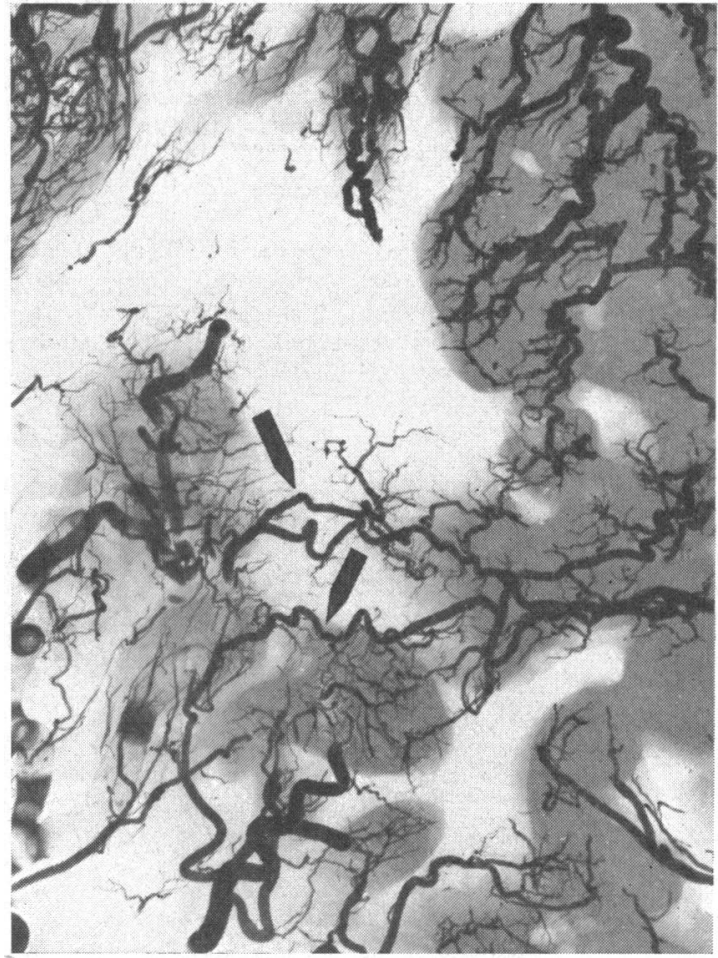

Fig. 11.-Communicating vessels between the left and right coronary artery trees are present, passing across the muscle columns of the anterior papillary muscle of the right ventricles. $(\times 4$.

by injection of a radiopaque medium, and the taking of microradiographs of transverse myocardial slices, is described.

The pattern of arteries in the right ventricle was found to be basically the same as that seen in the left ventricle, with both "branching" and "straight" type arteries being present. The possible importance of the blood supply to the right ventricle from the left coronary artery tree is discussed.

I am extremely grateful for the advice and encouragement of Professor W. B. Wartman, Professor George Dick, and Professor A. C. Thackray.

\section{REFERENCES}

Campbell, J. S. (1929). Stereoscopic radiography of the coronary system. Quart. F. Med., 22, 247.

Ehrich, W., de la Chapelle, C., and Cohn, A. E. (1931). Animal ontogeny, B. Man. I. A study of coronary arteries. Amer. F. Anat., 49, 241.

Farrer-Brown, G. (1967). The vascular supply of the myocardium of the ventricles of the human heart. M.D. Thesis, Cambridge. 
- (1968a). The injection of capillaries, arterioles, and arteries in the ventricles of the human heart by a radioopaque medium. Cardiovasc. Res., 2, 179.

- (1968b). Normal and diseased vascular pattern of myocardium of human heart. I. Normal pattern in the left ventricular free wall. Brit. Heart f., 30, 527.

Fulton, W. F. M. (1965). The Coronary Arteries. Thomas, Springfield, Illinois.

Gross, L: (1921). The Blood Supply to the Heart. Oxford University Press, London.

- and Kugel, M.A. (1933). The arterial blood vascular distribution to the left and right ventricles of the human heart. Amer. Heart f., 9, 165.
Horn, H., Field, L. E., Dack, S., and Master, A. M. (1950). Acute coronary insufficiency: pathological and physiological aspects: an analysis of 25 cases of subendocardial necrosis. Amer. Heart f., 40, 63.

Mitchell, J. R. A., and Schwartz, C. J. (1965). Arterial Disease, pp. 1-86. Blackwell, Oxford.

Wade, W. G. (1959). The pathogenesis of infarction of the right ventricle. Brit. Heart f., 21, 545.

Whitten, M. B. (1930a). A comparison of the blood supply of the right and left ventricles in childhood. Arch. intern. Med., 45, 46.

- (1930b). The relation of the distribution and structure of the coronary arteries to myocardial infarction. Arch. intern. Med., 45, 383. 